\title{
Prologue to Sparsity Issue
}

\section{Albert Cohen · Ronald A. DeVore • Michael Elad • Anna Gilbert}

Published online: 18 November 2008

(C) Birkhäuser Boston 2008

Some of the most important scientific challenges at the beginning of this century center on the understanding of complex processes arising in various area such as biosystems, climate modeling, or the internet. These challenges include not only the development of accurate mathematical models, but also the ability to compute and analyze the large data sets describing these processes. Fortunately, nature helps in that it is widely held that the description of these processes possesses a certain sparsity that helps bring some simplicity and understanding, provided we can discover and exploit what form this sparsity takes in a given setting.

The last decade has seen great clarification in what sparsity means and how it can be utilized in real problems. Mathematically, sparsity can be expressed in many ways but the central one is that the solutions to applied problems have a few dominant terms if they are captured in the correct representation system. This has motivated the

\footnotetext{
A. Cohen

Laboratoire Jacques-Louis Lions, Université Pierre et Marie Curie, 175, rue du Chevaleret, 75013 Paris, France

e-mail: cohen@ann.jussieu.fr

R.A. DeVore ( $₫)$

Department of Mathematics, University of South Carolina, Industrial Mathematics Institute,

Columbia, SC 29208, USA

e-mail: devore@math.sc.edu

M. Elad

Computer Science Department, The Technion-Israel Institute of Technology, Haifa 32000, Israel e-mail: elad@cs.technion.ac.il
}

A. Gilbert

Department of Mathematics, University of Michigan, 2074 East Hall, 530 Church Street, Ann Arbor, MI 48109, USA

e-mail: annacg@umich.edu 
development of new representation systems such as wavelets and their descendants, frames, dictionaries, and other redundant families. The challenge is not only to discover new representation systems which are better adapted for the sparse description of a given process, but also to develop analytical and numerical tools to capture the inherent sparsity when it occurs in such a representation. Thus, mathematical areas such as nonlinear approximation, compressed sensing, frames, convex optimization, and greedy algorithms have come to the forefront as major research themes related to sparsity. The field remains very active with new topics and applications continuing to emerge.

Since much of the mathematical development of sparsity takes place in the domain of Fourier Analysis and is directed at specific application areas, it is appropriate that the Journal of Fourier Analysis and Applications plays a leading role in representing the activity in this field. It is hoped that this special issue on sparsity will not only give a good representation of the state of the art of this emerging mathematical discipline but also lead to its further development. 